\title{
Coulomb Blockade and Insulator-to-Metal Quantum Phase Transition
}

\author{
Dmitri S. Golubev and Andrei D. Zaikin \\ Forschungszentrum Karlsruhe, Institut für Nanotechnologie, 76021 Karlsruhe, Germany \\ I.E.Tamm Department of Theoretical Physics, P.N.Lebedev Physics Institute, Leninskii pr. 53, 117924 Moscow, Russia
}

\begin{abstract}
We analyze an interplay between Coulomb blockade and quantum fluctuations in a coherent conductor (with dimensionless conductance $g \gtrsim 1$ ) attached to an Ohmic shunt. We demonstrate that at $T=0$ the system can be either an insulator or a metal depending on whether its total resistance is larger or smaller than $h / e^{2} \approx 25.8 \mathrm{k} \Omega$. In a metallic phase the Coulomb gap is fully suppressed by quantum fluctuations. We briefly discuss possible relation of this effect to recent experiments indicating the presence of a metal-insulator phase transition in $2 \mathrm{~d}$ disordered systems.
\end{abstract}

It is well known that Coulomb interaction may strongly affect quantum transport of electrons in mesoscopic conductors. For instance, electron tunneling across metallic junctions can be strongly suppressed or even blocked completely at $T=0$ due to Coulomb effects [1] 3]. This Coulomb blockade of tunneling is a direct consequence of the electron charge discreteness, manifestations of which persist even for junctions with resistances well below the quantum resistance unit $R_{Q}=h / e^{2} \approx 25.8 \mathrm{k} \Omega$.

Recently it was argued [4 that the $I-V$ curve of an arbitrary - albeit relatively short - coherent conductor in the presence of interactions can be expressed in the form

$$
R \frac{d I}{d V}=1-\beta f(V, T)
$$

where $1 / R=\left(2 e^{2} / h\right) \sum_{n} T_{n}$ is the Landauer conductance of a scatterer and $T_{n}$ are the transmissions of its conducting modes. The magnitude of the interaction term in eq. (11) is controlled by the parameter

$$
\beta=\frac{\sum_{n} T_{n}\left(1-T_{n}\right)}{\sum_{n} T_{n}},
$$

and $f(V, T)$ is a universal function which depends on $R$ as well as on the external impedance $Z_{S}(\omega)$ (e.g. leads) attached to the scatterer. A similar result was also obtained in Ref. [5] in the limit of a single conducting mode, in which case $\beta=1-T_{1}$.

The parameter (2) is already well known in the theory of shot noise [6]. Since shot noise and interaction effects in mesoscopic conductors are both the manifestations of the electron charge discreteness, it appears quite natural that the magnitude of both these effects is governed by the same parameter $\beta$ (2).

It is also important that the above effects do not disappear even if the charge quantization is not preserved in a part of the system (e.g. in the leads). Consider an important example of an Ohmic external impedance $Z_{S}(\omega) \simeq R_{S}$ and define $g_{S}=R_{Q} / R_{S}$ and $g_{0}=g+g_{S}$. The function $f(V, T)$ was already evaluated before for the case of tunnel junctions. At not very high energies $\max (T, e V) \ll g_{0} E_{C}$ and for $g_{0} \gg 1$ one finds

$$
f(V, T) \simeq\left(2 / g_{0}\right) \ln \left(g_{0} E_{C} / \max (T, e V)\right),
$$

i.e. in this regime Coulomb interaction causes a logarithmic correction to the scatterer conductance. This correction is small in the parameter $1 / g_{0}$. Here $E_{C}$ is the effective charging energy which sets the energy scale for the interaction effects in our problem. The result (3) is valid down to energies $\max (T, e V) \sim g_{0} E_{C} \exp \left(-g_{0} / 2\right)$. At even lower $T$ and $e V$ energy relaxation effects turn out to be particularly important making the conductance saturate at a universal value [4]

$$
G=\frac{1-\beta}{R}=\frac{2 e^{2}}{h} \sum_{n} T_{n}^{2},
$$

This formula recovers complete Coulomb blockade in the limit $\beta \rightarrow 1$ (tunnel junctions), demonstrates the absence of it for ballistic scatterers $(\beta \rightarrow 0)$, and yields suppression of the Landauer conductance by the factor $2 / 3$ in the important case of diffusive conductors.

Within the approach [4] one can demonstrate that all corrections to eq. (4t) are small in the parameter $\beta / g_{0} \ll 1$. Nevertheless, one cannot yet conclude that the system behavior is metallic at $T=0$ because the Langevin equation analysis [4] does not fully account for the charge discreteness in our system. Specifically, this analysis does not include subtle nonperturbative effects which can be captured only by means of the instanton technique. These effects may provide a mechanism which turns a conductor into an insulator at $T=0$. Thus, in order to understand the ground state properties of a system with disorder and interactions, the instanton effects should be properly taken into account.

In this Letter we will analyze the role of the instanton effects in relatively short coherent conductors. An important part of the work was already carried out by Nazarov [7] who found the renormalized Coulomb gap for a coherent conductor in the form $\tilde{E}_{C} \propto E_{C} \exp (-a g)$, where $a=\pi^{2} / 8$ for diffusive conductors [7,, 8$]$ and $a=1 / 2$ for tunnel junctions [9]. At temperatures below $\tilde{E}_{C}$ the 
charge quantization plays a crucial role and should yield insulating behavior at $T=0$. We will extend the instanton analysis [7] in several respects. Our main goal is to study the effect of an external impedance $Z_{S}$ on the renormalized Coulomb gap $\tilde{E}_{C}$. In particular, we will demonstrate that for $R_{S} \leq R_{Q}$ quantum fluctuations yield complete suppression of the Coulomb gap $\tilde{E}_{C}$ and, hence, destroy the insulating state even at $T=0$.

The model and effective action. As in Ref [4] we will consider an arbitrary coherent scatterer between two big reservoirs. Phase and energy relaxation are only allowed in the reservoirs and not during scattering, i.e. the scatterer is assumed to be shorter than both dephasing and inelastic relaxation lengths. Coulomb effects in the scatterer region are described by an effective capacitance $C$. The charging energy $E_{C}=e^{2} / 2 C$ is assumed to be smaller than the typical inverse traversal time.

The grand partition function $\mathcal{Z}$ of the system "scatterer+environment" and the scatterer effective action $S_{0}$ can be written in the form [2,10,11]

$$
\begin{gathered}
\mathcal{Z}=\sum_{l=-\infty}^{\infty} \int_{0}^{2 \pi l} \mathcal{D} \varphi \int \mathcal{D} q \exp \left[-\int d \tau \frac{C \dot{\varphi}^{2}}{2 e^{2}}-S_{0}[\varphi]+i \int d \tau \frac{\dot{\varphi} q}{e}-\frac{T}{2} \sum_{\omega}|\omega| Z_{S}(-i \omega)\left|q_{\omega}\right|^{2}\right], \\
S_{0}[\varphi]=\sum_{m=1}^{\infty} \frac{D_{m} T^{2 m}}{m} \int_{0}^{1 / T} d \tau_{1} \int_{0}^{1 / T} d \tau_{2} \ldots \int_{0}^{1 / T} d \tau_{2 m} \frac{\sin \left[\left(\varphi\left(\tau_{1}\right)-\varphi\left(\tau_{2}\right)\right) / 2\right]}{\sin \left[\pi T\left(\tau_{1}-\tau_{2}\right)\right]} \ldots \frac{\sin \left[\left(\varphi\left(\tau_{2 m}\right)-\varphi\left(\tau_{1}\right)\right) / 2\right]}{\sin \left[\pi T\left(\tau_{2 m}-\tau_{1}\right)\right]} .
\end{gathered}
$$

Here $\dot{\varphi}(\tau) / e=V(\tau)$ and $\dot{q}=I(\tau)$ are respectively the fluctuating voltage across the scatterer and the current through the system. Eq. (6) was initially derived for perfectly transparent contacts [10] and generalized in Ref. [11] to arbitrary transparencies. One has

$$
D_{m}=\frac{\mathcal{A} p_{F}^{2}}{2 \pi} \int_{0}^{1} \theta d \theta D^{m}(\theta)=\sum_{n}\left(1-R_{n}\right)^{m},
$$

where $\mathcal{A}$ is the cross section of the contact and $D(\theta)$ is its angle-dependent transparency. The sum $\sum_{n}$ is taken over independent conducting channels and $R_{n}=1-T_{n}$.

Isolated scatterer. Let us first consider an isolated scatterer $1 / Z_{S} \rightarrow 0$ and rederive the result [7] for $\tilde{E}_{C}$ from our effective action (6). In this limit integration over $q$ in (5) fixes the charge to be constant $i \int d \tau(\dot{\varphi} q / e) \rightarrow 2 \pi i l q_{x} / e$. At large conductances $g \gg 1$ the remaining integral over $\varphi$ is evaluated within the saddle point approximation. We are interested in the nontrivial saddle points $\tilde{\varphi}(\tau)$ for the action $S_{0}$ which "connect" states with different winding numbers $l$. A general expression for such instantons is given in [7] and in a certain limit it reduces to a set of Korshunov's instantons 12$] \tilde{\varphi}(\tau)=2 \arctan \left(\Omega\left(\tau-\tau_{0}\right)\right)$. Since the saddle point action $S_{0}[\tilde{\varphi}]$ does not depend on $\Omega$, it suffices to set $\Omega \rightarrow 0$. Then $\tilde{\varphi}$ reduces further to a set of "straight lines" $2 \pi T \tau l[13$. Substituting $\tilde{\varphi}(\tau)=2 \pi T \tau$ into (6) one trivially gets

$$
S_{0}[\tilde{\varphi}(\tau)]=\sum_{m=1}^{\infty} \frac{D_{m}}{m}=-\sum_{n} \ln R_{n} .
$$

Combining this equation with $\tilde{E}_{C} / E_{C} \propto \exp \left(-S_{0}[\tilde{\varphi}(\tau)]\right)$ one arrives at the result [7] (for a spin degenerate case).

It is also possible to go beyond the exponential accuracy and to estimate the pre-exponent in the expression for $\tilde{E}_{C}$. Here we restrict ourselves to an approximation of non-interacting instantons. Within this approximation $\tilde{E}_{C}$ can be found by means of a simple formula 14

$$
\left.\tilde{E}_{C} \sim T\left(\prod_{k=1}^{N} \frac{L_{k}}{z_{k}}\right)\left(S_{0}[\tilde{\varphi}(\tau)]\right)\right)^{N / 2} \exp \left(-S_{0}[\tilde{\varphi}(\tau)]\right),
$$

which yields correct results up to an unimportant numerical prefactor of order one. Here $L_{k}$ and $z_{k}$ are respectively the effective volume and the effective instanton size for the $k$-th zero mode and $N$ is the total number of zero modes. Similarly to [9] in our problem each instanton has two zero modes, corresponding to shifts of its center $\tau_{0}$ in time from zero to $1 / T$ and to fluctuations of its frequency $\Omega$ within the interval $0 \leq \Omega \lesssim E_{C}$ (fluctuations with $\Omega>E_{C}$ are exponentially suppressed by the charging term in the action). Thus we have $N=2, L_{1}=1 / T$ and $L_{2} \sim E_{C}$. The parameters $z_{1,2}$ for both zero modes can be easily evaluated. Substituting the eigenfunctions for these zero modes $\partial \tilde{\varphi} / \partial \tau$ and $\partial \tilde{\varphi} / \partial \Omega$ into the effective action one finds $z_{1} \sim 1 / \Omega$ and $z_{2} \sim \Omega$. Thus, the product $z_{1} z_{2}$ is $\Omega$-independent and just reduces to a numerical factor of order one $z_{1} z_{2} \sim 1$. Then eq. (9) immediately yields

$$
\tilde{E}_{C} / E_{C} \sim\left[\prod_{n} R_{n}\right] \ln \left[\prod_{n} R_{n}^{-1}\right] \sim a g \exp (-a g) .
$$

This formula is valid for $a g \gg 1$, i.e. either at large conductances $g \gg 1$ or, if $g \sim 1$, for very small values $R_{n}$ implying $a \gg 1$. In a (spin-degenerate) single channel limit eq. (10) reduces to $\tilde{E}_{C} / E_{C} \sim R_{1} \ln R_{1}^{-1}$ in agreement with the result derived in Ref. 15 for $R_{1} \ll 1$ within a different technique.

In order to find the dependence of the ground state energy $E_{0}$ on $q_{x}$ it is necessary to sum over all possible instanton configurations. For non-interacting instantons this summation yields 


$$
E_{0}\left(q_{x}\right)=-\Delta \cos \left(2 \pi q_{x} / e\right), \quad \Delta \sim \tilde{E}_{C} .
$$

In the particular case of a tunnel junction in the strong tunneling limit $g \gg 1$ this result agrees with one derived in eq. (10) of Ref. [9], where the pre-exponent in the expression for $\Delta$ (simply proportional to the inverse $R C$-time) was obtained by means of an explicit calculation of the fluctuation determinants. We also note that (comparatively weak) inter-instanton interaction might slightly modify the result (11), both the form of $E_{0}\left(q_{x}\right)$ and the pre-exponent in the expression for $\Delta$. However this effect will not be important for us here.

Now we are in a position to study the scatterer conductance at extremely low energies. In the limit of high external impedances $Z_{S} \gg R_{Q}$ both the resistance $R(\omega)$ and conductance $G(\omega)=1 / R(\omega)$ of our scatterer are determined from the correlation function $\langle\varphi \varphi\rangle$ evaluated for $1 / Z_{S} \rightarrow 0$. The calculation is again performed in imaginary time and is completely straightforward. Similarly to Ref. [16] let us make a shift $q_{x} \rightarrow q_{x}+\xi(t)$ in the exponent of eq. (5) and define the phase-phase correlator

$$
\langle\varphi(\omega) \varphi(-\omega)\rangle=-\left(\frac{1}{\omega^{2} \mathcal{Z}} \frac{\delta^{2} \mathcal{Z}}{\delta \xi_{\omega} \delta \xi_{-\omega}}\right)_{\xi=0} .
$$

In order to evaluate the generating functional $\mathcal{Z}[\xi(t)]$ (5) for $a g \gg 1$ it is sufficient to consider small fluctuations of $\varphi$ and instantons. Since we are mainly interested in the low frequency behavior of the correlator (12) we can safely assume that typical frequencies of the source field $\xi_{\omega}$ do not exceed the instanton ones $\Omega$. Under this adiabaticity condition our previous instanton analysis can be trivially repeated. Then for $q_{x} \rightarrow 0$ from eq. (12) we obtain

$$
\langle\varphi(\omega) \varphi(-\omega)\rangle=\frac{e^{2} R_{e f f}}{|\omega|}+\frac{4 \pi^{2} \Delta}{\omega^{2}}
$$

Here $\omega$ is the Matsubara frequency. What remains is to define the "Matsubara resistance" $\tilde{R}(\omega)=$ $\left(|\omega| / e^{2}\right)\langle\varphi(\omega) \varphi(-\omega)\rangle$ and perform an analytic continuation to real frequencies. After that at $T=0$ one gets

$$
G(\omega)=\frac{1}{R_{e f f}+(2 \pi / e)^{2} \Delta / i \omega} .
$$

This result implies that - while at $\omega \gg \Delta$ the conductance of our system remains finite - in the opposite low frequency limit $\omega \ll \Delta$ the renormalized Coulomb gap $\Delta \sim \tilde{E}_{C}$ becomes important, the response is predominantly capacitive and $G(\omega)$ vanishes at $\omega \rightarrow 0$. At nonzero but low $T \ll \Delta$ and $1 / Z_{S} \rightarrow 0$ one has $G(T) \propto \exp (-\Delta / T)$, i.e. the scatterer response is insulating at $T=0$ due to Coulomb blockade. A simple estimate of the effective resistance $R_{e f f}$ in (13), (14), similarly to [16], would give $R_{\text {eff }} \simeq R$. In the spirit of our Langevin equation analysis [4], one can also expect a (frequency dependent) correction to this estimate. In particular, at low $\omega<g E_{C} \exp (-g / 2)$ it is natural to expect $R_{\text {eff }} \simeq R /(1-\beta)$.

Metal-Insulator phase transition. Now let us see how the above behavior is modified in the presence of a finite external impedance $Z_{S}(\omega)$. In this case the charge $q$ fluctuates and, hence, should be treated as a quantum variable. For our purposes it is convenient to first perform the path integral (5) over the phase $\varphi(t)$. As before, in the limit $a g \gg 1$ we integrate over small fluctuations of $\varphi$ and instantons. Integrating out small fluctuations, for $|\omega| \lesssim E_{C}$ one arrives at an effective impedance

$$
\tilde{Z}(-i \omega) \simeq Z_{S}(-i \omega)+R,
$$

i.e. the scatterer impedance should simply be added to one of the shunt. Evaluating the instanton contribution we again assume that typical frequencies $\omega_{q}$ for the charge variable $q$ do not exceed $\Omega$. This is sufficient for $Z_{S} \gg R$. Then, similarly to [9], we find

$$
\mathcal{Z}=\int \mathcal{D} q \exp \left(-\frac{T}{2} \sum_{\omega}|\omega| \tilde{Z}(-i \omega)\left|q_{\omega}\right|^{2}-\int d \tau E_{0}(q)\right) .
$$

Let us now choose $Z_{S}$ to be Ohmic $Z_{S}(\omega) \simeq R_{S}$. In this case the resulting effective action for the charge $q$ coincides with one for a linearly damped quantum particle in a periodic potential (11). This is a well-known problem 17,2 which can be treated, e.g., by means of the standard renormalization group (RG) technique. Successively reducing the high frequency cutoff $\omega_{c}$ and integrating out charges with higher $\omega_{q}$ one arrives at the RG equations 17 for $\tilde{\Delta}=\Delta / \omega_{c}$ and $g_{\Sigma}=g g_{S} / g_{0}$ :

$$
\frac{d \tilde{\Delta}}{d\left(\ln \omega_{c}\right)}=\tilde{\Delta}\left(g_{\Sigma}-1\right)+O\left(\tilde{\Delta}^{3}\right), \quad \frac{d g_{\Sigma}}{d\left(\ln \omega_{c}\right)}=0 .
$$

Eqs. (16) demonstrate that for $g_{\Sigma}<1$ the value $\tilde{\Delta}-$ though initially small - grows in the course of renormalization. Thus the Coulomb gap remains finite and the system is an insulator at $T=0$. On the other hand, for $g_{\Sigma}>1$ the Coulomb gap scales to zero. In this case Coulomb blockade is completely destroyed by quantum fluctuations and the system behavior should be metallic down to $T=0$.

The charge-charge correlation function can also be derived in a straightforward manner. At $|\omega| \lesssim E_{C}$ one finds

$$
\langle q q\rangle_{\omega} \simeq\left(\left(R+R_{S}\right)|\omega|+\pi^{2} \Delta_{r} / e^{2}\right)^{-1} .
$$

Here $\Delta_{r}$ plays the role of the renormalized Coulomb gap. It is equal to $\Delta_{r}=\Delta\left(\Delta / \omega_{c}\right)^{\frac{g_{\Sigma}}{1-g_{\Sigma}}}$ for $g_{\Sigma}<1$ and $\Delta_{r}=0$ otherwise. As expected, charges are localized in the insulating phase and delocalized in the metallic one.

Choosing the initial cutoff frequency as $\omega_{c 0} \sim E_{C}$, one can rewrite the first eq. (16) directly for the combination $a g=\sum_{n} \ln R_{n}^{-1}$ : 


$$
d(a g) / d\left(\ln \omega_{c}\right)=\left(1-g_{\Sigma}\right)(1+1 / a g) .
$$

This equation is valid as long as $a g \gg 1$. In the tunneling limit $T_{n} \ll 1$ one has $\sum_{n} \ln R_{n}^{-1} \simeq \sum_{n} T_{n}=g / 2$, and (18) reduces to the RG equation for the tunneling conductance derived in [9] as well as in Refs. [18,19] (for the case $\left.g_{\Sigma}=0\right)$.

Combining our present results with those of Ref. [arrive at the following picture. In the limit of large conductances $g_{0} \gg 1$ the $I-V$ curve of an arbitrary coherent scatterer is described by eqs. (1)-(4) down to exponentially low temperatures and voltages $\max (T, e V) \gtrsim \Delta_{r}$. In this regime Coulomb effects lead to partial suppression of the scatterer conductance which saturates at the value (4) at low $T$ and $V$. For $g_{\Sigma} \geq 1$ or if at least one of the channels is ballistic, $R_{n}=0$, the Coulomb gap is fully suppressed $\Delta_{r}=0$, and the conductance remains nonzero (雨) even at $T=0$. This is the metallic phase. On the other hand, if $g_{\Sigma}<1$ and all $R_{n}>0$, at energies

$$
\max (T, e V) \lesssim \Delta_{r} \sim E_{C}[a g \exp (-a g)]^{\frac{1}{1-g_{\Sigma}}}
$$

Coulomb interaction leads to further suppression of the scatterer conductance $G$ which eventually vanishes at $T=0$. This is the insulating phase. A quantum phase transition between these two phases occurs either at $g_{\Sigma}=1$ or if the conductance (per spin) of at least one of the channels $g_{n} \equiv T_{n}$ becomes exactly equal to one.

Summarizing, an interplay between charge discreteness, coherent scattering and Coulomb interaction yields two types of effects. One effect is controlled by the parameter $\beta$ (2) and results in partial suppression of the system conductance at low $T$. For $g_{0} \gg 1$ this suppression is fully captured by the quasiclassical Langevin equation approach 4 . This effect of the electron-electron interaction is relatively robust in the sense that the interaction correction $\beta f$ (1) is never suppressed unless all the channels are ballistic $(\beta=0)$ and/or the shunt conductance $g_{S}$ is infinite. Another effect is due to instantons which give rise to the renormalized Coulomb gap $\Delta_{r}$. This interaction effect is much more subtle since the Coulomb gap can be destroyed by quantum fluctuations much easier. It is this suppression which yields a quantum insulator-to-metal phase transition in our system.

Finally, let us briefly address possible implications of our results for recent experiments [20] which strongly indicate the presence of a metal-insulator phase transition in various $2 \mathrm{~d}$ disordered systems. One can consider a (sufficiently small) coherent scatterer with the dimensionless conductance $g$ viewing all other scatterers in the system as an effective environment with the conductance $g_{S}$. On a phenomenological level one can assume this environment to be Ohmic at sufficiently low frequencies. Under this assumption one immediately arrives at the conclusion about the presence of a quantum metal-insulator phase transition at $g_{\Sigma}=1$. In 2 d systems one has $g \sim g_{S} \sim g_{0} \sim g_{\Sigma}$. Therefore in such systems this phase transition should be expected at conductances $\sim 1 / R_{Q}$, exactly as it was observed in numerous experiments [20]. Local properties of the insulating and metallic phases are expected to be very different. In the insulating phase charges should be localized around inhomogeneities (puddles) due to Coulomb blockade, while in the metallic phase the Coulomb gap is destroyed by quantum fluctuations and the charge distribution should be much more uniform. These expectations are fully consistent with recent experimental observations [21]. Thus, there might be a direct relation between the experimental results 22,21] and the old problem [17] describing dissipative dynamics of a quantum particle in a periodic potential. In order to explore this possibility a more detailed theory, which would include, e.g., the issue of quantum decoherence, would be warranted.

[1] D.V. Averin and K.K. Likharev, in Mesoscopic Phenomena in Solids, edited by B.L. Altshuler, P.A. Lee, and R.A. Webb (Elsevier, Amsterdam, 1991), p. 173.

[2] G. Schön and A.D. Zaikin, Phys. Rep. 198, 237 (1990).

[3] Single Charge Tunneling, edited by H. Grabert and M.H. Devoret, NATO ASI Series B, vol. 294 (Plenum, New York, 1992).

[4] D.S. Golubev and A.D. Zaikin, cond-mat/0010493.

[5] A. Levy Yeyati et al., cond-mat/0102178.

[6] Ya.M. Blanter and M. Büttiker, Phys. Rep. 336, 1 (2000).

[7] Yu.V. Nazarov, Phys. Rev. Lett. 82, 1245 (1999).

[8] A. Kamenev, Phys. Rev. Lett. 85, 4160 (2000).

[9] S.V. Panyukov and A.D. Zaikin, Phys. Rev. Lett. 67, 3168 (1991).

[10] G. Schön and A.D. Zaikin, Phys. Rev. B 40, 5231 (1989). A misprint in the prefactor in eq. (21) of this paper was corrected in eq. (C17) of Ref. [2].

[11] A.D. Zaikin, Physica B 203, 255 (1994).

[12] S.E. Korshunov, JETP Lett. 45, 434 (1987).

[13] R. Fazio and G. Schön, Phys. Rev. B 43, 5307 (1991).

[14] D.S. Golubev and A.D. Zaikin, cond-mat/0012104.

[15] K.A. Matveev, Phys. Rev. B 51, 1743 (1995).

[16] A.D. Zaikin and S.V. Panyukov, Phys. Lett. A 183, 115 (1993).

[17] A. Schmid, Phys. Rev. Lett. 51, 1506 (1983); S.A. Bulgadaev, JETP Lett. 39, 315 (1984); F. Guinea, V. Hakim, and A. Muramatsu, Phys. Rev. Lett., 54, 263 (1985); M.P.A. Fisher and W. Zwerger, Phys. Rev. B 32, 6190 (1985).

[18] F. Guinea and G. Schön, Europhys. Lett. 1, 585 (1986).

[19] W. Hofstetter and W. Zwerger, Phys. Rev. Lett. 78, 3737 (1997).

[20] For recent review see E. Abrahams, S. V. Kravchenko, M. P. Sarachik, Rev. Mod. Phys. 73, 251 (2001).

[21] S. Ilani et al. Phys. Rev. Lett. 84, 3133 (2000); A. Yacoby (private communication). 